\title{
Hibiscus extract, vegetable proteases and Commiphora myrrha are useful to prevent symptomatic UTI episode in patients affected by recurrent uncomplicated urinary tract infections
}

\author{
Tommaso Cai ${ }^{1}$, Daniele Tiscione ${ }^{1}$, Andrea Cocci $^{2}$, Marco Puglisi ${ }^{1}$, Gianmartin Cito ${ }^{2}$, \\ Gianni Malossini ${ }^{1}$, Alessandro Palmieri ${ }^{3}$ \\ ${ }^{1}$ Department of Urology, Santa Chiara Regional Hospital, Trento, Italy; \\ ${ }^{2}$ Department of Urology, University of Florence, Florence, Italy; \\ ${ }^{3}$ Department of Urology, University of Naples, Federico II, Naples, Italy.
}

\begin{abstract}
Summary Objective: To evaluate the efficacy of a combination of Hibiscus extract, vegetable proteases and Commiphora myrrha extract in the prophylaxis of symptomatic episode in women affected by recurrent urinary tract infections (rUTIs).

Materials and methods: In this phase II clinical trial, all patients with history and diagnosis of rUTI were enrolled. All patients underwent the following treatment schedule: 1 tablet in the morning and 1 tablet in the evening for 7 days and, then, 1 tablet in the evening for 10 days (1 cycle every each month, for 6 months) of a combination of Hibiscus extract, vegetable proteases and Commiphora myrrha extract. At the baseline, all patients underwent urologic visit with quality of life ( $2 o L$ ) questionnaires and mid-stream urine culture. After 3 and 6 months, all patients underwent urologic visit, urine culture and QoL questionnaires evaluation.

Results: Fifty-five women were enrolled (mean age 49.3; range: 28-61). At the enrollment time, the most common pathogen was Escherichia coli (63.7\%). The median number of UTI per 6 months was 5 (IQR: 4-9). At the end of the second follow-up evaluation, 25 women did not reported any symptomatic episode of UTI (49\%), 18 reported less than 2 episodes $(35.3 \%)$, while 8 reported more than 2 episodes (15.7\%). However, at the first and second follow-up evaluation the clinical statistically significant improvement (QoL) was reported by $38 / 51$ $(74.5 \%)(p<0.001$ from baseline) and 43/51 (84.3\%) $(p<$ 0.001 from baseline) women, respectively. The median number of UTI decreased to 2 (IQR: 0-3). At the end of the follow-up period, 30/51 had sterile urine (58.8\%), while 21/51 (41.2\%) reported a transition from symptomatic UTI to asymptomatic bacteriuria.

Conclusions: In conclusion, this treatment, in motivated patients, is able to prevent symptomatic UTI symptomatic episode and improve patient's QoL.
\end{abstract}

KEY WORDS: Urinary tract infection; Ellirose; Protelix; Serrazimes; Myrliq; Plant extracts; Antibiotic stewardship; treatment.

Submitted 11 August 2018; Accepted 24 August 2018

\section{INTRODUCTION}

Urinary tract infections (UTIs) represent the most frequently reported community-acquired infection and carry a significant burden for patients' quality of life and healthcare costs (1). Overall, more than 50\% of women report having had at least one UTI at some point in their lifetime, and it is estimated that almost $11 \%$ of women aged over 18 suffer from a UTI each year (2-3). In particular, UTIs recur approximately in 20-30\% of women within 6 months after their first episode $(1,4)$ and in around 35 to $53 \%$ of women within 12 months (5). The management of recurrent uncomplicated UTIs in women is still object of great discussion. To date, there are not well-established recommendations for a 'standard' prophylactic protocol to prevent recurrent UTI (6). Since the mainstay for the management of recurrent UTI still remains a short course of oral antimicrobial therapy, on the other hand the frequent use of antibiotics has led to the spread of multidrug-resistant microorganisms (710). Possible side effects, although rare, increasing healthcare costs, and rising bacterial resistance have progressively marked the downfall of classic antibiotic prophylaxis and, over years, paved the way to consider alternative prophylactic methods (4). In this regard nonpharmacological oral supplements, including the phytotherapy and nutraceuticals, seem to be an attractive alternative to antibiotic therapy to ameliorate symptoms related to UTIs and lower the rate of symptomatic recurrences. These considerations led us to conduct a prospective non-randomized phase II clinical trial in order to evaluate the efficacy of a combination of Ellirose $^{\circledR}$, Protexil $^{\circledR}$, Serrazimes ${ }^{\circledR}$ and Myrliq ${ }^{\circledR}$ (AVIUR ${ }^{\circledR}$ retard) in the prophylaxis of symptomatic episode in women affected by recurrent UTIs focusing on both the capability to reduce the number of symptomatic recurrences and the efficacy in improving QoL.

\section{Materials AND MEthods}

Study design

From September to December 2017, we enrolled in a prospective, non-randomized phase II study, all women affecting by recurrent uncomplicated UTI and attending our Centre. Written informed consent was obtained 
Figure 1.

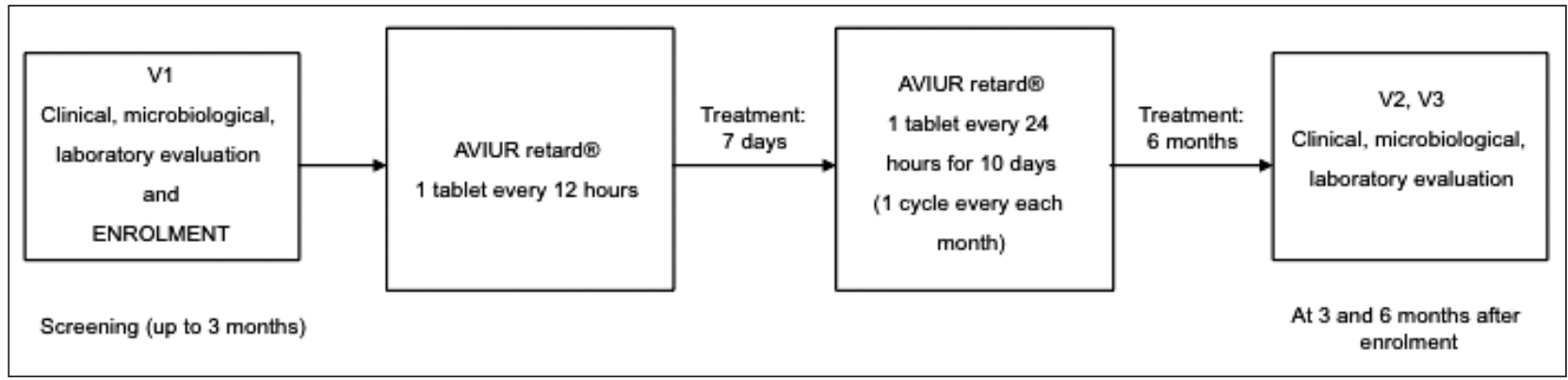

from all subjects before proceeding with the enrollment. Preliminary evaluation was performed by administering QoL questionnaires and by performing a preliminary mid-stream urine culture, in line with Cai $T$ et al. (11). Before enrolment all patients were treated with antibiotics in line with trialist's choice in order to obtain the infection free condition at baseline. The last isolated bacterial strain before the antibiotic treatment was collected and consider for this analysis. The main outcome measure was, then, the recurrence-free condition at the follow-up visit. All enrolled patients underwent the following treatment schedule: AVIUR retard ${ }^{\circledR} 1$ tablet in the morning and 1 tablet in the evening for 7 days and, then, 1 tablet in the evening for 10 days ( 1 cycle each month, for 6 months). After 30 days from the beginning of the treatment, all patients have been contacted by phone in order to ensure compliance to the treatment. Subsequent follow up schedule included urologic visit with QoL questionnaires and mid-stream urine culture performed after 3 and 6 months from the beginning of the therapy. In case of acute symptomatic UTI episode all patients were treated with antibiotics in line with European Association of Urology (EAU) guidelines on urological infections (12) and in line with the antibiogram.

The Figure 1 shows the study schedule.

\section{Clinical and microbiological considerations}

In accordance with the EAU guidelines, the diagnosis of UTI was defined according to the following parameters: patient reported symptoms and patient interview, physical examination, bed-side dip-stick urinalysis and urine culture (12). All microbiological and laboratory analyses have been described previously (13). In brief, all cleancatch midstream urine samples collected at room temperature were immediately taken to the laboratory under refrigerated conditions and analyzed. All urine samples were analyzed for common bacteria and yeasts, aliquoted for DNA extraction and polymerase chain reaction testing for Chlamydia trachomatis, Neisseria gonorrhoeae, and urogenital Mycoplasma. Microbiological culture was performed according to the procedure described by Hooton et al. (14). All other microbiological procedures and DNA extraction have been performed in line with Mazzoli et al. (15). For microbiological diagnosis, a colony count $\geq 105$ units $/ \mathrm{mL}$ was considered the cutoff for significant bacterial growth.

\section{Inclusion and exclusion criteria}

We included in this study all patients with recurrent
UTI, defined as $\geq 2$ infections in six months or $\geq 3$ infections in one year. Relevant exclusion criteria comprised: evidence of overactive bladder; anatomical abnormalities or previous surgery of the urinary tract; complicated UTI; pregnancy; urinary catheterization. Moreover, we excluded all patients with active infection by STD pathogens. We did not include any other exclusion criteria in order to make the results clinically relevant. Composition and characterization of the extracts used All patients who were treated in line with the manufacturer's instructions (Anatek Health Italia s.r.l. Via A. Caroncini, 5 - 20137 Milano; www.anatekhealth.com). Each administration contained a combination of $200 \mathrm{mg}$ Ellirose $^{\mathrm{TM}}, 100 \mathrm{mg}$ Protexil $^{\circledR}$ SP750, $100 \mathrm{mg}$ Serrazimes $^{\circledR}$, 60 mg Myrliq ${ }^{\circledR} P W D$.

\section{Outcome measures and statistical analysis}

The primary outcome of this study was the rate of recurrence at the end of the study period. A good response to treatment was defined as a significant reduction of recurrences ( $<2$ in six months). Treatment failure was defined as the absence of recurrence reduction at the end of the study period. Transition from symptomatic UTI to asymptomatic bacteriuria was not considered as treatment failure. In order to obtain significant results to analyze, sample size calculation was based on the following assumptions: difference in terms of recurrence between baseline and follow-up visit: $-3 \pm 1$; $\alpha$ error level, 0.05 two-sided; statistical power, $80 \%$; anticipated effect size, Cohen's $d=0.5$. The calculation yielded 43 individuals . Taking into account a drop-out rate of $10 \%$, the final sample size has been set to 49 patients in the both groups. Statistical analysis was performed by using SPSS.

\section{RESULTS}

Fifty-five women were enrolled (mean age 49.3; range: 28-61). At the time of enrollment, the most common isolated pathogen was Escherichia coli (63.7\%). All patients obtained sterile urine after antibiotic before enrollment. The median number of UTI per 6 months was 5 (IQR 49). Demographic, microbiological and clinical characteristics at the time of enrollment are reported in Table 1.

\section{Clinical and microbiological data at the first follow-up evaluation (3 months)}

After 3 months from the beginning of the treatment, 43 out of 51 patients $(84.3 \%)$ reported a clinical improvement in terms of QoL form the baseline $(p<0.001)$, 
Table 1.

Patient's sociodemographic anamnestic, clinical characteristics at enrolment time.

\begin{tabular}{|lc}
\hline No. of total patients & 55 \\
\hline Median age (range) & $49.3(28-61)$ \\
\hline Educational level & - \\
Primary school & $25(45.5)$ \\
Secondary school & $30(54.5)$ \\
Post-secondary education & $48(87.3)$ \\
\hline Sexually active (past month) & \\
\hline Current smoker & $21(38.2)$ \\
No & $34(61.8)$ \\
Yes & $49(89.1)$ \\
\hline Number of sex partners & $6(10.9)$ \\
1 & $39(70.9)$ \\
$\geq 1$ & $16(29.1)$ \\
\hline Number of UTI in the past 6 months & $35(63.7)$ \\
2 & $20(36.3)$ \\
$\geq 2$ & $35(63.7)$ \\
\hline Hormonal status & $10(18.2)$ \\
Pre-menopausal & $3(5.4)$ \\
Post-menopausal & \\
\hline Isolated strains before ABT treatment & \\
Escherichia coli & \\
Klebsiella spp. & \\
Enterococcus spp. & \\
Others & \\
\hline
\end{tabular}

while 42 out of 51 patients $(82.3 \%)$ reported a restore to pre-UTI situation. From a microbiological point of view, 40 patients (78.4\%) showed sterile urine and 11 (20\%) showed a transition from UTI to ABU (from Escherichia coli to Enterococcus Faecalis).

\section{Clinical and microbiological data at the second follow-} up evaluation ( 6 months)

At the end of the second follow-up evaluation, 25 women did not reported any acute episode of UTI (49\%), 18 reported less than 2 episodes (35.3\%), while 8 reported more than 2 episodes (15.7\%).

However, at the first and second follow-up evaluation the clinical statistically significant improvement (QoL) was reported by 38/51 (74.5\%) ( $p<0.001$ from baseline) and 43/51 (84.3\%) ( $p<0.001$ from baseline) women, respectively. Moreover, the median number of UTI decreased to 2 (IQR 0-3). From a microbiological point of view, at the end of the follow-up period, 30/51

\section{Table 2.}

All patient's sociodemographic anamnestic, clinical characteristics at enrolment time. ABT: antibiotic treatment.

\begin{tabular}{|lccc|}
\hline Outcomes variable & Baseline & $\begin{array}{c}\text { Follow-up } \\
\mathbf{1 8 0} \text { days }\end{array}$ & P \\
\hline Clinical improvement & & & \\
$\quad$ No episode of UTI & $0 / 51$ & $25 / 51(49)$ & \\
<2 episodes of UTI & $0 / 51$ & $18 / 51(35.3)$ & $<0.001$ \\
$>2$ episodes of UTI & $51 / 51$ & $8 / 51(15.7)$ & \\
\hline QoL (questionnaire) & 91.3 & 99.0 & $<0.001$ \\
\hline Microbiological improvement & & & \\
Sterile urine & $0 / 51$ & $30 / 51(58.8)$ & $<0.001$ \\
Transition to ABU from UTI & $0 / 51$ & $21 / 51(41.2)$ & $<0.001$ \\
\hline
\end{tabular}

sterile urine samples (58.8\%) and 21/51 (41.2\%) reported a transition from symptomatic UTI to ABU.

The Table 2 shows all clinical and microbiological data at the second follow-up evaluation.

Adherence results and treatment related adverse effects At the end of the study period, the adherence to the therapy was very high.

Only one patient (1.8\%) discontinued the prescribed therapy because of the onset of drug-related adverse effects, nausea in this particular case.

\section{Discussion}

The inappropriate use of antibiotics during last years has been paralleled by the growing onset of multidrug-resistant pathogenic strains among community-acquired isolates. The evolution of antimicrobial resistances has resulted into a global public health challenge, with rising costs and greater risk of poorer patient outcomes. Nowadays antibiotic stewardship protocols represent a precise imperative need. Unfortunately, to date there is not a "gold standard" prophylactic management to prevent recurrent UTI. Actually, European Association of Urology guidelines report several non-antibiotic measures for preventing recurrent UTIs but only a few are based on well-designed randomized clinical trials (13). However it cannot be denied that during last years research in non-antibiotic prophylaxis of recurrent UTIs has gradually grown. Particularly the use of phytotherapy and nutraceuticals might represent a feasible alternative approach for reducing the use of antibiotics and decreasing the rate of symptomatic recurrences $(4,16-$ 17). In this light, in this phase II clinical trial we evaluated the efficacy and safety of a new nutraceutical agent named containing Ellirose ${ }^{\circledR}$, Protexil ${ }^{\circledR}$, Serrazimes ${ }^{\circledR}$ and Myrliq ${ }^{\circledR}$ in the prophylaxis of symptomatic episodes in women affected by recurrent UTIs. In our experience AVIUR $^{\circledR}$ retard was well tolerated, resulting in optimal compliance since only one patient experienced adverse drug related effect which forced the discontinuation of the therapy. Moreover a statistically significant improvement in QoL was reported in almost $74.5 \%$ of patients and in $84 \%$ at first and second follow up visit respectively. Also from a microbiological point of view AVIUR ${ }^{\circledR}$ retard showed promising results since almost 60\% of patients at the end of the follow-up period showed sterile urine and more than $40 \%$ reported a transition from symptomatic UTI to ABU. To this regard, a significant body of evidence advocated that ABU could play a protective role in preventing symptomatic UTI recurrences, interfering with the establishment of many enteric pathogens, such as E. coli (8). Noteworthy the fact that AVIUR ${ }^{\circledR}$ retard is able to establish an $\mathrm{ABU}$ is a tangible proof of the absence of its effects on the normal microbiota. In this sense, the use of non-antibiotic approach should be preferred since it doesn't interfere with the normal commensal bowel flora. AVIUR ${ }^{\circledR}$ retard is made of a combination of several nutraceutical agents playing different roles in the prevention of symptomatic UTI recurrences. Ellirose ${ }^{\circledR}$, an Ibiscus dry extract, is a phytocomplex containing Sambubiosides, which seem to have an anti-inflammatory activity by reducing the levels of 
inflammatory mediators such as iNOS, NO, IL-6, MCP1 , and TNF- $\alpha$ induced by LPS (18). Protexil ${ }^{\circledR}$ is a form of concentrated protease derived as an exogenous production from controlled fermentation of maltodextrins through the Aspergyllus oryzae.

The protease, once absorbed into the blood stream, effectively performs anti-inflammatory and anti-edema functions (19-20). Particularly, the proteases from Protexil $^{\circledR}$ are bound to alpha 2-macroglobulines, which are thus modified into an activated form. Once activated, the alpha-2-macroglobulines bind the citokines TNFalpha (tumor necrosis factor alpha) and TGF-beta (transformative growth factor beta), modulating their activity in an anti-inflammatory way. Similarly, Serrazimes ${ }^{\circledR}$ is a proteolytic blend composed of enzymes derived from non-pathogenic enterobacteria belonging to genus Serratia species E-15. Serrazimes ${ }^{\circledR}$ contributes to the maintenance of a balanced immune system response by regulating protein-based immune cell inflammation mediators. It seems that Serrazimes ${ }^{\circledR}$ has also an additional analgesic activity due to its capability to block the release of biogenic amines. (19) Finally, Myrliq ${ }^{\circledR}$ is a Commiphora myrrha extract with a standardized content of curzerene, furanoeudesma-1,3-diene, and lindestrene and a high total furanodiene content (20) Recent evidence showed that myrrh extract inhibits the production of interluchin-6 (IL-6) and interluchin-8 (IL-8) in human fibroblast cells. In addition it is involved in the blockage of the proteins involved in the inflammatory process such as Cox and also inhibits the formation of NO, ROS, TNF- $\alpha$, PGE2, NF-kB and MAPK (21-22). Our results showed that AVIUR $^{\circledR}$ retard can be taken into consideration as a potential effective prophylactic agent for symptomatic recurrent UTIs. However, the present study was not devoid of several limitations. Possible shortcomings of the study lie in the lack of randomization as well as in the small cohort size. Each of these variables might have introduced statistical bias and weaken the overall reliability of the reported findings. Acknowledged the limitations, the present study represents the largest series so far evaluating the prophylactic effect of Ellirose ${ }^{\circledR}$, Protexil $^{\circledR}$, Serrazimes $^{\circledR}$ and Myrliq ${ }^{\circledR}$ in women suffering from recurrent uncomplicated UTIs. Although antimicrobials remain the mainstay of treatment for acute UTI, the spread of multidrug resistant microorganisms among community-acquired isolates is worryingly increasing and needs for stronger surveillance and new preventing approaches. Only advancing our understandings about alternative treatments, such as phytotherapty and nutraceuticals, will enable us to develop appropriate non-antibiotic approaches and prevention strategies for recurrent UTIs. To this regard, further phase III randomized studies and larger series will certainly be needed to confirm and validate our preliminary results.

\section{Conclusions}

Recurrent urinary tract infections afflict a large number of young women nowadays, with a high impact on patient's quality of life. Currently, several strategies have been purposed and used in order to reduce the number of recurrence and improve QoL, without any significant achievement.

Here, considering the available evidence, the use of phytotherapy seems a feasible antibiotic-sparing approach for reduce the number of recurrence.

In our experience, the treatment with AVIUR retard ${ }^{\circledR}$, in motivated patients, is able to prevent symptomatic UTI episode and improve patient's QoL. Moreover, the use of AVIUR retard ${ }^{\circledR}$ is be able to improve the antibiotic stewardship, reducing the use of antibiotic prophylaxis in these patients. However, further prospective studies are needed to validate our results in larger series.

\section{References}

1. Silverman JA, Schreiber HL 4th, Hooton TM, Hultgren SJ. From physiology to pharmacy: developments in the pathogenesis and treatment of recurrent urinary tract infections. Curr Urol Rep. 2013; 14:448-56.

2. Foxman B, Brown P. Epidemiology of urinary tract infections: transmission and risk factors, incidence, and costs. Infect Dis Clin North Am 2003; 17:227-241.

3. Jennifer A. Silverman, Ph.D., Henry L. et al. From physiology to pharmacy: developments in the pathogenesis and treatment of recurrent urinary tract infections. Curr Urol Rep. 2013; 14:448-56.

4. Cai T, Tamanini I, Kulchavenya E, et al. The role of nutraceuticals and phytotherapy in the management of urinary tract infections: What we need to know? Arch Ital Urol Androl. 2017; 89:1.

5. Bojana Kranjčec, Dino Papeš, Silvio Altarac. D-mannose powder for prophylaxis of recurrent urinary tract infections in women: a randomized clinical trial. World J Urol. 2014; 32:79-84.

6. Tandogzu Z, Wagenlehner FM. Global epidemiology of urinary tract infections. Curr Opin Infect Dis. 2016; 29:73-79.

7. Kostakioti M, Hultgren SJ, Hadjifrangiskou M. Molecular blueprint of uropathogenic Escherichia Coli virulence provides clues toward the development of anti virulence therapeutics. Virulence. 2012; 3:592-594.

8. Cai T, Mazzoli S, Mondaini N, et al. The role of asymptomatic bacteriuria in young women with recurrent urinary tract infections: to treat or not to treat? Clin Infect Dis. 2012; 55:771.

9. Gupta K, Scholes D, Stamm WE. Increasing prevalence of antimicrobial resistance among uropathogens causing acute uncomplicated cystitis in women. JAMA 1999; 281:736-8.

10. Naber KG, Bergman B, Bishop MC, et al. Urinary Tract Infection (UTI) Working Group of the Health Care Office (HCO) of the European Association of Urology (EAU). EAU guidelines for the management of urinary and male genital tract infections. Eur Urol. 2001; 40:576-88.

11. Cai T, Cocci A, Tiscione D, et al. L-Methionine associated with Hibiscus sabdariffa and Boswellia serrata extracts are not inferior to antibiotic treatment for symptoms relief in patients affected by recurrent uncomplicated urinary tract infections: Focus on antibiotic-sparing approach. Arch Ital Urol Androl. 2018; 90:97-100.

12. Grabe M, Bjerklund-Johansen TE, Botto $H$ et al. Antibiotics. In:Grabe M, Bjerklund-Johansen TE, Botto $H$, et al. (Editors) Guidelines on urological infections. Arnhem: European Association of Urology. 2010; p. 69.

13. Cai T, Nesi G, Mazzoli S, et al. Asymptomatic bacteriuria treatment is associated with a higher prevalence of antibiotic resistant 
strains in women with urinary tract infections. Clin Infect Dis. 2015; 61:1655-61.

14. Hooton TM, Scholes D, Gupta K, et al. Amoxicillin-clavulanate vs ciprofloxacin for the treatment of uncomplicated cystitis in women: a randomized trial. JAMA. 2005; 293:949-55.

15. Mazzoli S, Cai T, Rupealta V, et al. Interleukin 8 and antiChlamydia trachomatis mucosal IgA as urogenital immunologic markers in patients with C. trachomatis prostatic infection. Eur Urol. 2007; 51:1385-93.

16. Stange R, Schneider B, Albrecht U, et al. Results of a randomized, prospective, double-dummy, double-blind trial to compare efficacy and safety of a herbal combination containing Tropaeoli majoris herba and Armoraciae rusticanae radix with cotrimoxazole in patients with acute and uncomplicated cystitis. Res Rep Urol. 2017; 9:43-50.

17. Ledda A, Belcaro G, Dugall M, et al. Highly standardized cranberry extract supplementation (Anthocran ${ }^{\circledR}$ ) as prophylaxis in young healthy subjects with recurrent urinary tract infections. Eur Rev Med Pharmacol Sci. 2017; 21:389-393.
18. Sogo T, Terahara N, Hisanaga A, et al. Anti-inflammatory activity and molecular mechanism of delphinidin 3-sambubioside, a Hibiscus anthocyanin. Biofactors. 2015; 41:58-65.

19. Bhagat S, Agarwal M, Roy V. Serratiopeptidase: a systematic review of the existing evidence. Int J Surg. 2013; 11:209-17.

20. Germano A, Occhipinti A, Barbero F, et al. A pilot study on bioactive constituents and analgesic effects of MyrLiq ${ }^{\circledR}$, aCommiphora myrrha Extract with a High Furanodiene Content. Biomed Res Int. 2017; 2017:3804356

21. Su S et al. Evaluation of the anti-inflammatory and analgesic properties of individual and combined extracts from Commiphora myrrha, and Boswellia carterii.. J Ethnopharmacol. 2012; 139:649-56.

22. Bellezza I, et al. Furanodien-6-one from Commiphora erythraea inhibits the NF- $k B$ signalling and attenuates LPS-induced neuroinflammation. Mol Immunol. 2013; 54:347-54.

\section{Correspondence}

Tommaso Cai, MD

ktommy@libero.it

Daniele Tiscione, $M D$

Marco Puglisi, MD

Gianni Malossini, MD

Department of Urology, Santa Chiara Hospital

Largo Medaglie d'Oro 9, Trento, Italy

Andrea Cocci, MD

Gianmartin Cito, MD

Department of Urology, University of Florence, Florence, Italy

Alessandro Palmieri, MD

Department of Urology, University of Naples, Federico II, Naples, Italy 